\title{
A new use for statins?
}

Statins, the widely used cholesterol-lowering drugs, could also treat the disabling disease multiple sclerosis (MS), according to research in the 7 November issue of Nature. Scott Zamvil and colleagues used mouse models of MS to show that oral atorvastatin prevented or reversed chronic and relapsing paralysis.

There are five statins available at present: lovastatin, simvastatin, pravastatin, fluvastatin and atorvastatin. They inhibit the enzyme HMG-CoA reductase, which controls the rate of cholesterol production in the body. These drugs lower cholesterol by slowing down its production, and by increasing the ability of the liver to remove the harmful lowdensity lipoprotein (LDL) cholesterol already in the blood. Statins have been used for more than 20 years now, and are a cheap and relatively safe treatment for atherosclerosis and coronary heart disease. Studies have also shown that statins can inhibit the production of pro-inflammatory molecules.

MS is thought to arise when autoimmune helper $\mathrm{T}$ cells attack the myelin sheath of the spinal cord and neurons, causing

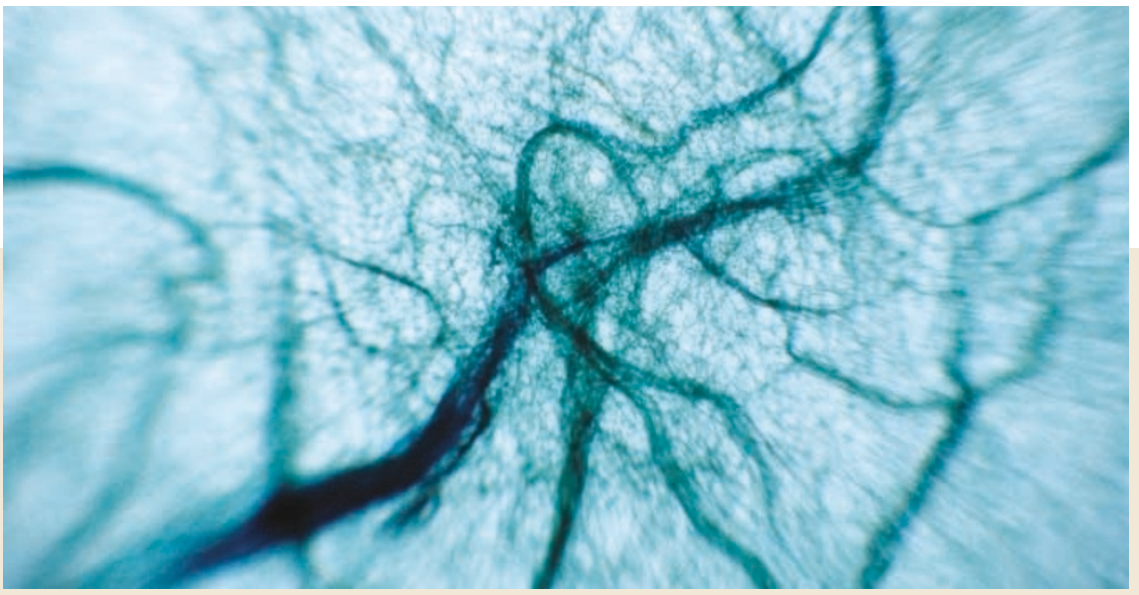

unpredictable symptoms, which range from fatigue and vision problems to paralysis. Current treatments include the use of interferon- $\beta$, which reduces the inflammation associated with nerve damage, or copaxone, a synthetic, basic, random co-polymer that is thought to inhibit the autoimmune attack. However, these treatments slow rather than stop the progression of the disease.

When tested in experimental autoimmune encephalomyelitis (EAE) mice, which are widely used as models for human MS, atorvastatin had a beneficial effect, preventing disease relapse and reducing inflammation in the nervous system. Atorvastatin seems to redirect myelinspecific helper $\mathrm{T}$ cells from a destructive autoimmune role to one of suppressing autoimmunity. A subset of helper T cells no longer produces inflammatory cytokines, but rather anti-inflammatory ones. Although cholesterol reduction could affect the behaviour of helper $\mathrm{T}$ cells, the authors have found another explanation for the effects described in their paper: atorvastatin inhibits the expression of a key immunesystem protein, the class II transcriptional activator (CIITA), which controls the expression of major histocompatibility complex (MHC) class II molecules. Without the expression of MHC class II molecules on certain immune cells, helper $T$ cells cannot be activated, and consequently, they are not destructive. It is not clear whether the reduction in CIITA expression is caused directly by atorvastatin, or is an indirect effect of reduced inflammation.

Statins are in clinical trials at present for human MS, and would make a cheap, orally available therapeutic for MS. However, results from EAE models are not always found to be reproducible in humans, and sometimes give the opposite outcome to that seen in man.

Melanie Brazil

(10) References and links ORIGINAL RESEARCH PAPER Youssef, S. et al. The HMG-CoA reductase inhibitor, atorvastatin, promotes a $\mathrm{T}_{\mathrm{H}} 2$ bias and reverses paralysis in central nervous system autoimmune disease. Nature 420, 78-84 (2002)

\section{PRION DISEASES}

\section{Toxic waste}

It is widely believed that an altered version of the cell-surface glycoprotein $\operatorname{PrP}$ - known as $\mathrm{PrP}^{\mathrm{Sc}}$ - has a pivotal role in transmissible prion diseases, such as the neurodegenerative disorder Creutzfeldt-Jakob disease. But, two

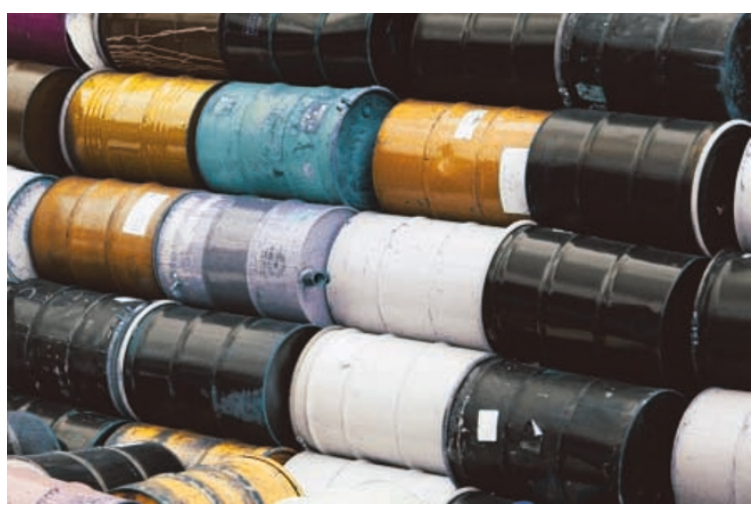

papers by Lindquist and colleagues in Science Express now show that the true culprit for neurotoxicity is, in fact, not $\mathrm{PrPsc}$

PrP begins the journey from folding to the cell surface in the endoplasmic reticulum (ER). A substantial proportion of PrP misfolds, and this is detected by the qualitycontrol mechanism, which transports the misfolded protein into the cytosol to be degraded by the proteasome.

In the first study, Ma and Lindquist showed that when proteasome activity is inhibited, cytosolic PrP accumulates and forms amorphous aggregates, and some of this converts to a PrPsc-like form.

So, which form is the killer? In the second study, Lindquist and colleagues found that it wasn't the conversion to $\operatorname{PrP}^{\mathrm{sc}}$ that led to neuron death, but rather the accumulation of $\operatorname{PrP}$ in the cytosol.

On the basis of their findings, Lindquist and colleagues have proposed a unifying model for PrP-associated diseases. A portion of PrP misfolds in the ER (this misfolding might be increased by disease-causing PrP mutations) and is retrogradely transported to the cytosol for proteasomal degradation. Normally, this misfolded PrP is degraded by the proteasome, but if the activity is compromised (for example, by stress or age), even small amounts of accumulated cytotoxic PrP can be neurotoxic. $\mathrm{PrP}^{\mathrm{Sc}}$ is not the toxic species, but pathogenesis could be caused by $\operatorname{PrP}^{\mathrm{sc}}$ affecting the folding and transport of PrP.

The findings have both good and bad implications. First, alterations in PrP transport that prevent toxic accumulation of $\operatorname{PrP}$ in the cytosol could be a potential therapeutic strategy for prion diseases. Second, because interfering with normal proteasome function leads to the accumulation of cytosolic PrP, proteasome-inhibitor therapies (which are being developed for cancer) might need to be treated with caution.

(2) References and links

Simon Frantz Original Research papers Ma, J \& Lindquist, $S$.

Conversion of PrP to a self-perpetuating PrPsc-like conformation in the cytosol. Science Express 2002 Oct 17 (doi: 10.1126/science.1073619) | Ma, J., Wollmann, R. \& Lindquist, S. Neurotoxicity and neurodegeneration when PrP accumulates in the cytosol. Science Express 2002 Oct 17 (doi: 10.1126/science.1073725) 